le portiQue Le Portique

Revue de philosophie et de sciences humaines

$5 \mid 2000$

Passages du siècle

\title{
L'attentat de Sarajevo
}

Maurice Gandillac (de)

\section{(2) OpenEdition}

Journals

Édition électronique

URL : http://journals.openedition.org/leportique/389

DOI : 10.4000/leportique.389

ISSN : $1777-5280$

Éditeur

Association "Les Amis du Portique"

Édition imprimée

Date de publication : 1 janvier 2000

ISSN : 1283-8594

Référence électronique

Maurice Gandillac (de), «L'attentat de Sarajevo », Le Portique [En ligne], 5 | 2000, mis en ligne le 24 mars 2005, consulté le 25 mars 2021. URL : http://journals.openedition.org/leportique/389 ; DOI : https://doi.org/10.4000/leportique.389

Ce document a été généré automatiquement le 25 mars 2021

Tous droits réservés 


\title{
L'attentat de Sarajevo
}

\author{
Maurice Gandillac (de)
}

1 Sarajevo - ou Serajevo (les deux se disent) -, au début de l'été 14, les «grandes personnes » n'ont à la bouche que ces quatre syllabes dont la sonorité évoque pour un petit Parisien de huit ans et demi le vieux Trocadéro en briques rouges et son exotisme de pacotille. À cette ville, le Nouveau Larousse illustré des années 1900 donne comme premier nom Bosna-Seraï. Installé jadis sur les rives de la «Bosna » par le conquérant de Byzance, Mohammed II, ce « sérail » n'est plus alors que le modeste chef-lieu (moins de 25000 habitants) d'une province, depuis quatre cents ans ottomane, où les « grandes puissances ", au Congrès de Berlin en 1878, chargèrent l'Autriche-Hongrie de rétablir l'ordre " au nom du sultan », mais qu'en 1908, deux ans après ma naissance, Vienne a tout simplement annexée, provoquant à Belgrade une très vive irritation.

2 De ces chaudes semaines de juillet 1914 (riches en violents orages), certes je garde le souvenir des baignades sur l'étroite plage de Guétary, de promenades à bicyclette, d'une excursion dans les Pyrénées et j'ai retrouvé un "cahier de brouillon » où je vantais les charmes de notre petit hôtel sans aucune mention des bruits de guerre. Ce n'est pourtant point par illusion rétrospective que je me remémore ce village basque entre le fronton de pelote et la voie ferrée où passent le soir les voitures jaunes paille du « Sud-Express » - sous la menace chaque jour aggravée d'un conflit plus grave que la série de guerres balkaniques, avec leurs successifs renversements d'alliances à peu près incompréhensibles pour un enfant.

3 Et voici qu'à nouveau, en ce temps même où j'entreprenais la rédaction de mes Mémoires, l'image d'un pont bosniaque s'est imposée sur nos écrans, plus présent que ne l'était, soixante-deux ans plus tôt, celui qu'on pouvait voir alors sur les photos du journal Excelsior, montrant le lieu où, le 28 juin 1914, un jeune "patriote » venait d'assassiner l'archiduc François-Ferdinand, ce prince héritier qui - dès la mort, présumée assez proche, du vieux François-Joseph couronné en 1848 - avait prévu, semble-t-il, une réorganisation fédérative de l'empire qui eût assuré aux Slaves, à côté des Allemands et des Magyars, une part légitime de pouvoir dans un ensemble bigarré mais uni par l'histoire et la géographie ${ }^{1}$. 
Dans ma petite enfance, concurrencée depuis peu par l'Entente cordiale (et son apport de nouvelles anglomanies), l'alliance de la république avec l'empire des tzars, pourvoyeuse de "montagnes russes" sur les manèges de nos foires, jouait encore en politique un rôle déterminant. Les propos des adultes et les articles de journaux (dont d'aucuns s'étonnaient de me voir déjà curieux, mais la mode était alors d'apprendre à lire dès trois ans d'âge) soulignaient la vocation naturelle des Russes à protéger des peuples apparentés. Je n'ai guère souvenir qu'on évoquât beaucoup la vieille et toujours brûlante coupure entre Rome et Byzance, quelque peu occultée par l'unité de langue (écrit en deux alphabets, le même serbo-croate est parlé par les fidèles de trois religions) ni qu'on soulignât la présence, dans l'ancien vilayet ottoman, d'une forte communauté d'anciens chrétiens, de longue date convertis à l'islam et que le Larousse illustré traitait simplement de « Turcs».

5 En ce début du $x^{e}$ siècle, sur mainte enseigne ou firme commerciale qualifié encore de «nouveau » avec le parfum de fraîcheur qui accompagne en général cet adjectif, les anciennes pratiques nées du fanatisme et de l'intolérance - anathèmes sur le vaincu qui, dans le meilleur des cas, n'échappait jadis à la mort que par la servitude, djihads, croisades, pogroms, purifications ethniques - semblaient bien raréfiées, sinon même révolues. Cependant, à Adama, selon les journaux du temps, on comptait, en mai 1909, trois mille cadavres d'Arméniens massacrés, préfiguration peut-être des prochaines horreurs, scientifiquement élaborées, qu'allaient connaître nos jeunes aînés, des balles dum-dum au «nettoyage » des tranchées et aux nappes asphyxiantes.

En juillet 14, l'Europe est, à certains égards plus civilisée que celle que nous connaîtrons ensuite; à la seule exception des russes et des turques, ses frontières se franchissent sans passeport et les identités se justifient par une simple enveloppe à votre nom ou l'attestation d'un concierge. Dans toutes les grandes capitales on joue à peu près les mêmes pièces, on écoute la même musique, on acclame les mêmes danseurs, on lit les mêmes romans, voire les mêmes traités de philosophie ${ }^{2}$. Héritage des siècles où tous les monarques (et, par bâtardise, beaucoup de leurs sujets) descendaient peu ou prou de Charlemagne, de saint Louis et de Charles Quint, proches parents sont, en cette veille de guerre, les trois souverains régnant de l'Irlande au Pacifique, George V et Nicolas II, qu'on prendrait presque pour des jumeaux, mais aussi leur cousin Guillaume II, ce monarque éclairé qu'un grand professeur français, arrière petit-fils d'un Juif venu de Bavière avant la Révolution française, fils du librettiste de La Belle Hélène, considérait au seuil du nouveau siècle comme "le plus intelligent des souverains d'Europe », seul capable d'unir l'« aristocratisme » à l'« industrialisme » et même au " socialisme » 3 .

7 Tutoyant King et Tzar, le Kaiser, de son yacht, leur enverra de fraternels messages cependant que les chancelleries des deux camps (sans excepter notre Quai d'Orsay) préparent, sinon "fraîchement et joyeusement ", du moins sans grand scrupule et de façon quasiment inconsciente, un carnage de quatre années pire sans doute que tous ceux qu'avait commis et subis jusqu'alors l'humaine inhumanité. Et dans trop de familles et d'écoles, par la voie aussi d'une mauvaise littérature " pour enfants », chez nous comme, à coup sûr, en maints autres pays, on suggère déjà aux jeunes lecteurs, face aux "ennemis héréditaires ", de façon brutale ou plus insidieuse, une foncière méfiance, parfois la haine et le mépris.

8 Certes, né vers le temps où Armand Fallières venait de remplacer Émile Loubet à l'Élysée ${ }^{4}$, mes souvenirs d'enfance remontent bien plus haut, aux omnibus à chevaux descendant du Panthéon vers un carrefour Médicis où un petit train à vapeur attendait 
devant le Luxembourg l'heure de partir pour Arpajon - l'été au paysan périgourdin ne parlant souvent que patois, presque analphabète, marchant pieds nus sur les chemins et dans les champs, n'ayant guère sur le dos et autour des hanches que ce qui me reste en mémoire comme des morceaux de sacs et chiffons -, prËs de la frontière d'Alsace (qui se franchit, comme partout tout en Europe, sans passeport ni autre pièce d'identité), aux nouvelles casernes s'élevant chaque année, au spectacle des " grandes manœuvres", officiers à cheval sur les hauteurs, jumelles en main, observant les fantassins en pantalon garance qui avancent en ligne, baïonnette au canon.

Sur un cahier de brouillon, qui contient des exercices scolaires de l'été $1913{ }^{5}$, je note, au crayon (maintenant à peine lisible), la description de l'horloge astronomique qu'un suisse chamarré présente successivement en allemand et en français. Au Hohneck où quelques bornes indiquent seules le partage de la montagne entre le Reichsland ElsaßLothringen et le département des Vosges, passent et repassent à leur guise, des deux côtés de la fameuse «ligne bleue " ${ }^{6}$, civils et militaires des deux nations. Aux pages suivantes le cahier mentionne, le 21 juillet 1913, l'arrivée «d'un aéroplane et d'un dirigeable; le 8 août le « départ de quatre biplans pour Nancy ». Apparaissaient même dans le ciel des triplans et des machines volantes de formes singulières, mais on gardait quelque tendresse pour ces dirigeables Clément Boyard, plutôt ventrus, qui s'efforçaient alors de concurrencer les longs « cigares » du comte Zeppelin.

Avant 14, quand on dit : "Un sou est un sou », on sait encore, de façon très vague, ce que signifia, il y a bien longtemps, le solidus latin. Avec le « petit sou» de bronze (cinq centimes), on achète un petit pain ou une tablette de chocolat ; c'est également le prix du timbre sur la carte postale. Pour une lettre (à coup sûr distribuée le lendemain), il faut un "gros sou» (dix centimes). C'est ce que coûtent aussi l'enregistrement de trente kilos de bagages dans le train et le ticket de métro (« trois sous » l'aller et retour acheté avant 9 h. du matin). La lourde «thune » de «cent sous » (cinq francs) dépasse largement le salaire journalier d'un ouvrier.

Il me souvient d'une leçon pratique sur le système métrique, donnée à domicile, un jour de petite maladie, par une jeune institutrice du cours privé de Neuilly que je fréquentais à partir de 1912. J'ai constaté ce jour-là que le volume d'une boîte de fer blanc contenant du «brouillon Kub » était exactement d'un décimètre cube, sa capacité d'un litre et que le poids de cette eau sur la balance de la cuisine, une fois déduite la tare, était bien d'un kilo. Si j'ai retenu cet épisode, c'est parce que le K de Kub paraissait alors fort suspect aux maniaques du chauvinisme. Déjà le bruit courait que, derrière chaque plaque murale vantant ce produit helvétique, les Allemands, pour guider leurs troupes, avaient inscrit de précieuses indications stratégiques. En 14, dès le jour de la mobilisation, la foule pillera les boutiques Maggi.

12 On le sait bien, en ces années de l'immédiat avant-guerre, ce qu'il est convenu d'appeler la «modernité » s'affichait, en maints domaines et franchissait toutes frontières. Connus d'un petit nombre, Freud et Einstein n'occuperont le devant de la scène, presque simultanément, qu'au lendemain de la Guerre et un enfant de mon âge n'en eût rien retenu, mais la « T.S.F. ", avec ses merveilleuses apparences d'action à distance et de quasi ubiquité, fascine les imaginations. On s'étonne seulement qu'elle n'ait pu prévenir le heurt d'un iceberg par un bateau flambant neuf. Du Titanic je dessine gauchement les quatre longues cheminées sur du gros papier marron (celui dont le boucher enveloppe alors la viande). Son naufrage, en avril 1912, gâte un peu ma confiance enfantine dans le progrès. De même les tâtonnements de la radiothérapie, 
cruelle pour ses pionniers. Alors que d'aucuns attendent du radium de vrais miracles, une des nièces par alliance de mon grand-père va mourir en quelques mois, victime de cette leucémie dont on m'explique le mécanisme en me décrivant un brusque pullulement de globules blancs.

13 Un peu décevantes aussi les applications de la « Fée Électricité » telles que les présente une «Maison » sur les grands Boulevards. On y voit des chambres à coucher modern style munies d'une table de nuit sur laquelle un monte-charge offre, au réveil, à portée de main, gazette du jour, café au lait et pain beurré. Sur la table de salle à manger, un tapis roulant apporte les plats de la cuisine et y ramène ensuite les assiettes sales, mais il n'est pas question de machines à laver la vaisselle et, faute de réfrigérateur, on se contentera longtemps de la glacière qu'approvisionnent encore des voitures à chevaux. Plus spectaculaire me paraît, sur la Place de l'Opéra, face au Café de la Paix, le Journal Lumineux où, par petites ampoules mobiles, s'inscrivent déjà les dernières nouvelles, mais à l'heure où s'impose le plus lourd que l'air, Robida, dans ses albums pour enfants, dessine des villes où circulent de fenêtre à fenêtre, les mêmes petits dirigeables que présente Georges Méliès dans des films trop vite oubliés.

Je ne crois pas avoir jamais entendu parler alors de Mondrian et de Kandinsky mais on fustige autour de moi une manière de peindre qui déforme choses et gens, les réduit à des cubes ou même ne veut plus rien «représenter». Déjà Debussy et Ravel surprennent (et choquent) les amateurs de Gounod et de Massenet. Que dire alors de la dodécaphonie dont Schönberg présente les règles en 1911 ? À la maison on s'intéresse pourtant aux Ballets russes et quand ma mère décrit les fantastiques bonds de Vaclav Nijinski dans l'Oiseau de feu et le Spectre de la rose, c'est pour moi grand dépit de n'être encore admis à ces festivités. Pour sa musique plus que pour sa chorégraphie, le Sacre du printemps va pourtant être hué.

15 En cette année 1913, qu'un demi-siècle plus tard le C.N.R.S. n'aura pas tort de choisir comme date charnière pour un volume collectif, je pressens que tout bouge et je devine très vaguement un renouveau auquel ont même contribué, à la fin du xIX $x^{\mathrm{e}}$ siècle encore si proche, le Paul Bourget des Essais de psychologie contemporaine (défricheur à sa façon de ces terrains encore peu cultivés: l'inconscient et l'âme russe) et le presque écologiste Maurice Barrès du Jardin de Bérénice, ouvrages, cela va sans dire, que je n'emprunterai que beaucoup plus tard à la bibliothèque paternelle.

16 Mais c'est de bien autre chose qu'on s'entretient à Guéthary, en cette fin de juillet 1914 où d'heure en heure l'atmosphère s'alourdit. Quand bat enfin le tambour du garde champêtre pour la lecture du fatal décret, qui peut encore croire que, comme l'affirme Viviani, la mobilisation ne soit pas la guerre, celle que l'Autriche-Hongrie, depuis deux jours, a si promptement déclarée à la Serbie et qui va s'étendre, en quatre années, aux cinq continents? Oubliant tout le mal que j'ai entendu dire de Poincaré, entraîné par les autres enfants dans une sorte de farandole patriotique aux cris répétés de "Vive la France! À bas l'Allemagne! Mort au Kaiser!» (prononcé Késer), si je ne joins que faiblement ma voix aux autres, c'est surtout par inaptitude naturelle (et durable) à toute manifestation chorale.

Un pensionnaire avec qui nous sympathisons décide de conduire aussitôt en voiture à Irun la fraülein de ses enfants et l'Allemande prend congé de leurs petits camarades de jeu avant le long voyage qui, par l'Espagne et l'Italie, lui permettra de rejoindre sa famille. Comme la chose la plus naturelle, à son gentil mot d'adieu j'oppose cette ferme déclaration: "Vous êtes maintenant mon ennemie " ${ }^{7}$. Beaucoup plus tard, lisant Aus 
meinem Leben, je saurai quels liens courtois et même amicaux se nouèrent, pendant la Guerre de Sept Ans, entre la famille Gœthe ${ }^{8}$ et le comte Thorane, distingué amateur d'art qu'un billet de logement installa chez eux, avec mission de faciliter les rapports entre l'armée et la population. Depuis le temps où les vainqueurs accueillaient à leur table, comme des pairs, les généraux vaincus, les mœurs ont fort changé (et les conditions objectives dont elles dépendent, mais qu'elles contribuent aussi à déterminer). En 14, de ces us et coutumes ne survivra guère que dans l'aviation naissante une règle non écrite de courtoisie, estime chevaleresque entre chasseurs qui sur des appareils fragiles s'affrontent à découvert, en combat singulier.

Quand, boulimique de tout imprimé ${ }^{9}$, je lis attentivement les trois affiches qu'on vient de coller sur le mur de la petite mairie, j'éprouve un sentiment de malaise à constater qu'au décret qui «mobilise » les Français en âge et en état de combattre s'en joignent deux autres qui «réquisitionnent", l'une les chevaux, mulets et voitures, l'autre les « indigènes " d'Algérie et de Tunisie, "sujets » et "protégés » d'une fausse "patrie " qui ne devient telle que lorsqu'il leur faut la «défendre» et mourir pour elle au « champ d'honneur ».

En quelques heures, bizarrement, le «billon " s'est raréfié. Pour l'enregistrement de notre lourde malle ventrue, un de ces jeunes hommes qui vont se faire faucher par les mitrailleuses de Charleroi passe à ma mère l'indispensable "gros sou ». À son remerciement stéréotypé : "Je vous les rendrai au Paradis!»j'entends encore l'immédiate réaction: «Non, madame, à Berlin!». Croyait-il vraiment que nos pioupious bousculeraient d'un coup l'armée impériale et, franchis Rhin et Elbe, défileraient bientôt Unter den Linden? Dans les moments de ferveur patriotique, pareils slogans servent d'anesthésiques.

La suite, on la connaît : la débâcle de 1870 (comme, d'avance, celle de 1940) évitée de si peu grâce au courage des fantassins qui sans panique reculent de la Meuse à la Marne (avant l'auto les gens de chez nous savaient marcher), la faute stratégique de l'envahisseur infléchissant sa route vers l'ouest, sa provisoire pénurie de munitions et l'art de Galliéni l'attaquant sur son flanc, la course à la mer, l'enfouissement dans des tranchées continues, de la Manche à la frontière suisse et, de part et d'autre, les vains coups de boutoir pour réussir l'impossible "percée ", - Clemenceau, jour après jour, dans L'Homme libre, rappelant que « les Allemands sont à Noyon"-, les Zeppelins, les Gothas et la Grosse Bertha (l'obus sur Saint-Gervais le Vendredi Saint 18) -, les impardonnables hécatombes du Chemin des Dames, l'apport salvateur des Américains, la paix manquée et les prémisses accumulées d'une nouvelle catastrophe, tout cela dérivant - au moins pour une part essentielle - de l'attentat de juin 14.

21 Au niveau le plus superficiel, signe pourtant d'une révolution des mœurs ${ }^{10}$, on va voir les femmes, en bien peu d'années, renoncer aux lourds chignons et aux grands chapeaux, aux abominables corsets et à l'entassement des jupons, se couper les cheveux et mettre au jour leurs bras et leurs jambes. Notables aussi la fin de valeurs aussi sûres que le trois pour cent de la rente française, la dévaluation du sterling, un gigantesque gaspillage et une affreuse saignée. Indéniables certes, (mais oserait-on parler de «compensation»?) le développement des nouvelles techniques et maintes transformations, souvent heureuses, dans les modes de vie, tout cela, bien sûr, favorisé par les besoins de la guerre et ensuite ceux de la reconstruction, mais en choisissant ici Sarajevo comme date symbolique, je pense surtout à quatre conséquences de grand poids, si peu contestables qu'il suffira ici de les rappeler d'un mot. 
D'abord une évidente régression spirituelle. Bien loin des trêves de Dieu et des guerres en dentelle, malgré la Croix-Rouge et la Convention de Genève, les hommes de mon âge ont vu renaître, reprise et aggravation du jeu napoléonien, la barbarie du combat à peu près permanent, combinant le «nettoyage » des tranchées, la destruction systématique des personnes et des biens, le mépris cultivé de l'adversaire, source de maints ressentiments.

Ensuite l'extension de la belligérance à plusieurs continents et, à travers le drame wilsonien (les fameux «Points» du Président, conditions si vite trahies de l'intervention américaine), la vocation naissante des États-Unis comme régente de l'Europe, esquisse d'une hégémonie qui ne s'imposera réellement qu'à la seconde Guerre.

24 En troisième lieu, non point là où Marx et Engels l'annonçaient mais aux marges de la vieille Europe dans une Russie si mal préparée à une guerre qu'avaient pourtant voulue plus d'un de ses dirigeants, mais si vite à bout de forces, le déclenchement d'un processus révolutionnaire qu'avant 14 Jaurès et ses amis imaginaient bien autrement, convaincus que les masses, par leur immédiate révolte, auraient d'abord rendu impossible le combat à mort entre les grandes puissances industrialisées. Nous n'avons pas fini de mesurer ce que signifient - suites directes d'une guerre épuisante dans une zone excentrique de l'Europe qui, sortant à peine du Moyen Âge, subissait déjà les rudes débuts du capitalisme - la mainmise bolcheviste sur la révolution et l'instauration par Lénine d'un nouveau despotisme (partiellement) éclairé, bien différent de la dictature du prolétariat telle que l'annonçait Marx, étape sans doute nécessaire, mais présumée provisoire, vers l'extinction de la puissance étatique.

5 Comme quatrième conséquence, entre bien d'autres, je retiens ici, parce qu'elle nous tient plus que d'autres à cœur, à côté de cette puissance nouvelle qui, de Cuba à Pékin, de Moscou à Pnom-Penh, va s'affirmer sur tous les continents et tendre partout des « rideaux » séparateurs -, en foncière rivalité avec elle mais non sans quelques fâcheux moments de connivence ${ }^{11}$-, après l'opérette mussolinienne combinant Machiavel et Marinetti ${ }^{12}$, l'ignoble vésanie hitlérienne, liée assurément à des théories biologiques venues et des pays voisins et du siècle précédent, tenant aussi et, plus lointainement, à de tenaces préjugés religieux, mais très directement issue des échecs et des rancœurs d'un combattant déçu de la première Guerre.

Certes on pouvait imaginer un autre Traité de Versailles, une plus attentive gestion des virtualités de Locarno, une meilleure approche des problèmes sociaux et de la nécessaire décolonisation. Il est permis de rêver un autre $\mathrm{xx}^{\mathrm{e}}$ siècle ou tenter de n'en retenir que quelques précieuses découvertes médicales et tout ce qui, par l'automatisation et l'électronique, a su - du moins dans une portion déjà privilégiée du globe - alléger très notablement la peine des humains. Qui oserait pourtant, au terme de ce siècle, confirmer l'imprudente anticipation de Hugo et le saluer comme la vraie fin de Satan? Interrogé sur les moments les plus heureux qu'il a vécus, non certes dans sa vie privée mais comme simple Français moyen, le nonagénaire que je suis largement en cette pénultième année du siècle (la dernière qui se désigne par le chiffre 19 encore si prometteur d'avenir pour l'enfançon que je fus en sa première décennie) - est certes tenté d'évoquer quelques journées de grande liesse ou de vive espérance, le 11 novembre 18 ou le 6 juin 44, mais ne serait-ce point occulter les moments les plus abominables de deux guerres mondiales, d'une révolution manquée et de leurs interminables suites : Verdun, le goulag, Auschwitz, Hiroshima, le Cambodge de Pol Pot 
et tant de combats sanglants qui, en ce printemps 99 , sévissent encore dans les mêmes Balkans qui connurent le meurtre de François-Ferdinand et le fatal ultimatum viennois?

Ce ne serait en tout cas que retenir quelque péripéties de l'événement majeur qui suivit de si près et par une terrifiante logique l'attentat de Serajevo, celui dont un garçon de huit ans vécut avec grande émotion l'annonce solennelle dans un petit village de notre côte basque: enthousiasme naïf assez vite retombé, vive perception des altérités, conscience encore vague d'un « moment » décisif du jeune $\mathrm{xx}^{\mathrm{e}}$ siècle.

De cette conjoncture on ne pourra enseigner, ce me semble, aux écoliers futurs qu'après un long délai (à supposer qu'alors il reste des hommes qui, sur une Terre vivable, aient encore envie de philosopher), dans quelle catégorie «historique » il est convenable de la situer, à côté de la captivité de Babylone, de Salamine ou d'Alésia, de Poitiers et de Lépante ou, beaucoup plus simplement, comme le début d'une des plus saugrenues parmi toutes les guerres civiles.

\section{NOTES}

1.. En mai 18, le Department of State imaginera un plan de ce genre, mais le président Wilson n'osera même pas le présenter à ses partenaires de Versailles en raison des promesses prodiguées en vrac par l'Entente aux Roumains, aux Polonais, aux Tchèques et aux Serbes.

2.. Malgré la vocation du Portique et ma qualité d'agrégé de philosophie (mais je ne me suis jamais voulu qu'historien d'idées et de doctrines), interrogé pour savoir quel fut à mes yeux l'événement marquant du siècle, jamais l'idée ne m'est venue de le choisir philosophique. Marx, Kierkegaard, Nietzsche, même Freud et Bergson sont des hommes du xIX ${ }^{e}$ siècle (comme en d'autres domaines Proust et encore Valéry et Claudel). Quoi qu'on pense de Husserl et de Heidegger et sans sous-estimer les philosophes de grand talent de ma propre génération ni davantage les plus jeunes que, durant ma longue carrière, j'ai vus passer au concours d'agrégation et devant des jurys de thèse (d'Althusser à Deleuze, de Foucault à Derrida et il en est bien d'autres de très haut niveau), il ne me semble pas que cette période ait été marquée ni par une personnalité ni par une œuvre faisant durablement époque. Les vraies révolutions du siècle ressortissent à la science et surtout à la technique.

3.. Formules que je découvrirai en 1996 dans la correspondance d'Élie Halévy publiée chez Fallois par François Furet. On y trouve curieusement, bien avant 40, appliqué aux Français (et même, de façon très surprenante, à ces Anglais dont l'auteur fut un historien attentif), le qualificatif gaullien de " veaux ».

4.. Trois semaines plus tard, le coup de grisou de Courrières provoquera une grève suivie d'une brutale répression. L'année précédente Combes a cédé la présidence du conseil à Rouvier, qui, pour négocier un compromis avec l'Allemagne sur le Maroc, s'est débarrassé de Delcassé, l'homme de l'Entente Cordiale. Picasso présente les Demoiselles 
d'Avignon, les « Fauves » s'imposent au Salon d'automne, Saussure inaugure son cours à Genève ; Bergson publie L'Évolution créatrice.

5.. Dans un « problème » d'arithmétique, il est question d'un kilo de savon coûtant 80 centimes, d'un litre de rhum payé 3 francs. Une autre fois on demande, sachant qu'une somme d'argent se compose de 10 pièces de 5 francs, 7 de 2 francs et 6 de 1 franc, quelle est cette somme et quel en est le poids (réponse correcte : « 70 francs qui pèsent 350 grammes »).

6.. Je lis dans de vieux numéros de L'Illustration qu'en août 1908 des officiers prussiens en uniforme ont participé à l'inauguration d'un monument allemand érigé à Mars-laTour en souvenir d'un épisode de la Guerre de 70. Un peu plus tard, pour l'érection d'un monument français, à Wissembourg, territoire annexé, des officiers français en uniforme furent à leur tour accueillis et l'on joua même la Marseillaise. Après 1911, plus vives deviennent les tensions. À Pâques 1919 quand nous retrouverons Saint-Dié, faisant par la même voie le même voyage à Strasbourg, cette fois il nous faudra présenter un «sauf-conduit » pour franchir ce qui n'est pourtant plus une frontière. Nous voici à présent, et pour longtemps, dans l'univers de la séparation et du soupçon. 7.. Sans avoir encore lu Horace, j'ai retrouvé d'instinct la trop fameuse réplique : « Rome vous a nommé, je ne vous connais plus ». Assez vite je regretterai cette sottise, avant même, je crois, d'apprendre au lycée la différence entre hostis et inimicus.

8. Certes divisée entre un grand-père attaché à l'empereur François de LorraineHabsbourg, allié provisoire des Français, et les plus jeunes que fascinait, sinon le Prusse, du moins Frédéric II, mais le futur séducteur de Frédérique Brion apprécia, nous dit-il, la douceur des desserts français et tous les Francfortois - après la bataille de Sondershausen, revanche du pauvre Soubise, ridiculisé à Rossbach - gâtèrent indistinctement les blessés welches et teutons arrivant mélangés dans les lazarets de la ville.

9.. Bientôt, tout en jouant au train dans la petite cour de mon grand-père, sur le carrelage où courent des cafards, avec des boites de sucre Say ou Béghin réunies par des ficelles, j'y entasserai par rubriques des articles découpés dans les journaux. 10.. Plus lent fut celle du jugement de valeur sur les structures familiales. Sans remonter au temps de Sade ni au Paris de Rétif de la Bretonne, les « outrages aux bonnes mœurs » n'étaient pas plus rares au début qu'en fin de siècle et l'on sait ce que fut, à cet égard, la « fin » du précédent, l'époque de Pierre Louys et de Jean Lorrain, mais outre-Manche on internait Oscar Wilde et notre préfecture de police veillait à ce que fussent bien clos les pantalons des danseuses de cancan. Dans ma jeunesse La Garçonne fit encore scandale ; aujourd'hui presque personne ne s'émeut qu'il ne sorte guère de film sans coït simulé et les parents les plus bourgeois admettent sans peine que leurs filles, usant de la " pilule ", vivent très innocemment en couples libres. 11.. La plus malencontreuse étant, bien sûr, celle de 39-41.

12.. À Lisbonne, avant Vichy et Bratislava, ce fascisme intégrera une dose appréciable de thomisme plus ou moins dévoyé. 


\section{RÉSUMÉS}

À partir du nom de Sarajevo, lieu de l'attentat d'où sortit la première Guerre Mondiale et qui devait reparaître quatre-vingts ans plus tard au premier plan de l'actualité, un nonagénaire, évoquant des souvenirs d'enfance, s'interroge sur les apports du siècle presque traversé. Face à un prodigieux bond en avant de la science et surtout de la technique, il craint que - d'Auschwitz à Hiroshima - trop d'abominations suggèrent tout le contraire d'un progrès spirituel. 